\title{
Coupling Analysis for the Thermo-Acoustic-Vibration Response of a Thin-Walled Box
}

\author{
Yan Yang and Chen-Wu Wu
}

\begin{abstract}
Monitor and control unit is essential to almost all of the modern aircrafts or spacecrafts, which always places in a relatively closed space and requires a compatible mechanical environment to ensure its normal function. The acoustic, vibration and thermal loads developed in the operation of the aircraft determine a complex environment for its monitor and control unit. In particular, there should be couple effect among the thermal, vibration and acoustic responses. This article presents numerical simulation studies on a thermo-acoustic-vibration response of a typical thin-walled box, to reveal the multi-physics environment of the monitor and control unit. The thermo-structural equations were proposed and solved numerically, following that the vibro-acoustic analysis was implemented. The coupled algorithm was developed to simulate the structure response and sound distribution, and predict the acoustic loss during transmitting through the box shell. The validation cases of the vibration and acoustic response of a plate were performed at first. The outcome provides the multi-physics environment prediction method for designing and optimizing the monitor and control unit.
\end{abstract}

Keywords Thermal - Vibration - Acoustic - Multi-physics environment

\section{Introduction}

Acoustic-vibration is an important coupled mechanical environment for modern aircrafts or spacecrafts, especially for the monitor and control unit, which comprises precision instruments and behaves sensitive to strong vibration and noise. In gen-

\footnotetext{
Y. Yang

LHD, Institute of Mechanics, Chinese Academy of Sciences, Beijing 100190

People's Republic of China

e-mail: yangyan@imech.ac.cn

C.-W. Wu $(\square)$

Institute of Mechanics, Chinese Academy of Sciences, No.15 Beisihuanxi Road,

Haidian District, Beijing 100190, People's Republic of China

e-mail: chenwuwu@imech.ac.cn
} 
eral, vibro-acoustic responses experiments should be performed in the design and development stages [1]. For decades, numerical simulation and prediction techniques have been developed increasingly to help the design and optimization of the mechanical environment of spacecrafts devices. As to vibro-acoustic analysis, coupled finite element method (FEM) and boundary element method (BEM) has been widely used, in order to reduce the computation cost.

As known, when the environmental temperature changes, the structure property will change, in particular the elastic modulus of the materials, which affects the material properties and vibro-acoustic behavior of the structure [2]. Not much work has been done in the thermo-vibro-acoustic analysis for multiple physics. Jeyaraj et al. [2] studied the vibration and acoustic response of a plate in a thermal environment subjected to a harmonic load, in which the thermal stress effect is included in a so called geometric stiffness matrix without considering the softening of the materials due to temperature elevation. Geng and Li [3] studied the vibration and acoustic radiation characters of an isotropic rectangular thin plate under thermal environments, in which the thermal structure stress is assumed to be the only contribution made by the thermal environment.

In this study, a thermo-vibro-acoustic analysis was implemented to investigate the multi-physics environment of a typical thin-walled box model, representing the monitoring and control unit of a spacecraft. Firstly, the thermo-structural equations were proposed and solved numerically with finite element method (FEM). Secondly, the vibro-acoustic analysis was made by using boundary element method (BEM).

\section{Methods}

The numerical simulation was performed, by using FEM and BEM through below procedure. In detail, the temperature and thermal stress are calculated by FEM for every thermal equilibrium state with considering the thermal softening of the materials. The vibration and acoustic response are computed by BEM for typical thermal equilibrium state.

\subsection{Thermo-Vibro-Acoustic Equation and Numerical Methods}

The equation of motion for a prestressed structure with the sound pressure load is as follows;

$$
[\mathbf{M}]\{\ddot{\mathbf{U}}\}+[\mathbf{C}]\{\dot{\mathbf{U}}\}+\left[\mathbf{K}+\mathbf{K}_{\sigma}\right]\{\mathbf{U}\}=\left\{\mathbf{F}^{\mathbf{t}}\right\}+\left\{\mathbf{R}_{s} \mathbf{p}\right\}
$$


where $[\mathbf{M}]$ is the inertial matrix, $[\mathbf{U}]$ is the displacement vector, $[\mathbf{C}]$ is the structural damping matrix, $[\mathbf{K}]$ is the temperature-dependent stiffness coefficient matrix, $\left[\mathbf{K}_{\sigma}\right]$ is the increment stiffness coefficient matrix induced by thermal stress, $\left[\mathbf{F}^{\mathrm{t}}\right]$ is the applied load vector (supposed time harmonic), $\mathbf{p}$ is the acoustic pressure, and $\mathbf{R}_{s}$ is the coupled matrix describing acoustic pressure acting upon the structure.

For harmonic solution when $\mathbf{F}^{\mathrm{t}}(t)=\mathbf{F}_{\mathrm{s}} e^{j \omega t}$, assuming $\mathbf{U}(t)=\mathbf{U} e^{j \omega t}$, the Eq. (1) turns into,

$$
\left(\left[\mathbf{K}+\mathbf{K}_{\sigma}\right]+j \omega \mathbf{C}-\omega^{2}[\mathbf{M}]\right)(\{\mathbf{U}\})+\mathbf{L}_{c}\{\mathbf{p}\}=\left\{\mathbf{F}_{\mathrm{s}}\right\}
$$

where $\mathbf{L}_{\mathrm{c}}$ is the vibro-acoustic coupled matrix, $\mathbf{F}_{\mathrm{s}}$ is the harmonic amplitude of the force vector.

The basic governing equation of sound wave is Helmholtz equation (in frequency domain), assuming the sound wave is harmonic,

$$
\nabla^{2} p-k^{2} p=-j \rho_{0} \omega q
$$

where $p$ is acoustic pressure, $k=\omega / c=2 \pi f / c$ is wave number, $\rho_{O}$ is the density of the fluid, and $q$ is the sound source. The boundary element method for acoustic problem is written as,

$$
\rho_{0} \omega^{2}[\mathbf{G}] \mathbf{u}_{\mathbf{f}}+[\mathbf{H}] \mathbf{p}=\mathbf{F}_{\mathrm{a}}
$$

where $\mathbf{H}$ and $\mathbf{G}$ are boundary integral influence matrices, $\mathbf{F}_{\mathrm{a}}$ is the sound source vector, $\mathbf{p}$ and $\mathbf{u}_{\mathrm{f}}$ are the acoustic pressure and displacement of the boundary element nodes of the fluid. The coupled FEM/BEM is implemented to solve the structuralacoustic problem, using ANSYS and SYSNOISE respectively. First the pre stressed harmonic analysis is performed in ANSYS, the result file is imported into SYSNOISE to find the acoustic-structural response, where the indirect harmonic BEM is used.

\subsection{The Model}

The model was set up as a thin-walled box, as Fig. 1 shows, with geometry $0.5 \times 0.3 \times 0.2 \mathrm{~m}^{3}$, with thickness $2.5 \mathrm{~mm}$. The material is specified as steel, which is herein modeled as isotropic and ideal elastic with Young's modulus $E=200 \mathrm{GPa}$, Poisson's ratio 0.3 , mass density $7800 \mathrm{~kg} / \mathrm{m}^{3}$, thermal expansion coefficient $1.0 \times 10^{5}$ under ambient temperature condition [4]. The acoustic medium is modeled as air, with density $1.21 \mathrm{~kg} / \mathrm{m}^{3}$ and sound speed $340 \mathrm{~m} / \mathrm{s}$. The fluid material properties are supposed to be temperature-independent in this study. Without loss of generality, one normally incident harmonic plane sound wave, with sound pressure level $140 \mathrm{~dB}$, is imposed upon the top of the box. In this study, there is no force excitation, in order to focus only on the acoustic environment. 
Fig. 1 The sketch of the model

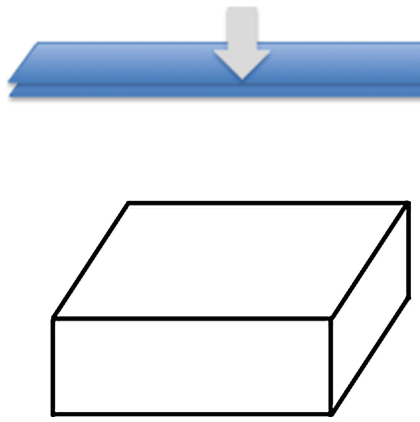

Two cases, denoted by 'cool' and 'hot', where two values of Young's modulus determined by the material temperature are specified, that is, in the hot case the material is supposed to has $70 \%$ Young's modulus than that of the cool one, i.e., $140 \mathrm{GPa}$ in magnitude. Such stiffness reduction is always related to the temperature elevation of the materials, for which the linear approximation of elastic modulus dependent on temperature is assumed [4].

The volume element mesh of FEM model is matched with the shell element mesh of BEM, where the shell element size is set as $10 \mathrm{~mm}$, and the thickness of volume element is equal to the thickness of the box, $2.5 \mathrm{~mm}$. Such mesh size can resolve the acoustic analysis up to $5666 \mathrm{~Hz}$.

\section{Results and Discussion}

The natural modes. The four corner points in the bottom wall plate of the box are fixed in computation. The first 25 modes were analysis with FEM. Table 1 shows the first 5 and the 25th natural frequencies of the box in cool and hot conditions. It is clear that the natural frequencies of the box reduce with increase in temperature. The first 2 mode shapes are shown in Fig. 2.

Vibro-acoustic response characteristics. The vibro-acoustic response may be represented by the acoustic field response at the center point of the box. Figure 3 is the sound pressure level at the center point $\left(S P L_{\text {center }}\right)$, and the transmission loss $(T L)$ here is defined as,

$$
T L_{(1)}=S P L_{\text {in }}-S P L_{\text {center }}
$$

Table 1 Natural frequencies of the box under different ambient temperature

\begin{tabular}{l|l|l|l|l|l|l}
\hline Natural frequencies (Hz) & Mode 1 & Mode 2 & Mode 3 & Mode 4 & Mode 5 & Mode 25 \\
\hline Cool & 179.3 & 299.0 & 391.5 & 394.8 & 435.9 & 1526.1 \\
\hline Hot & 150.0 & 250.1 & 327.5 & 330.3 & 364.7 & 1276.8 \\
\hline
\end{tabular}



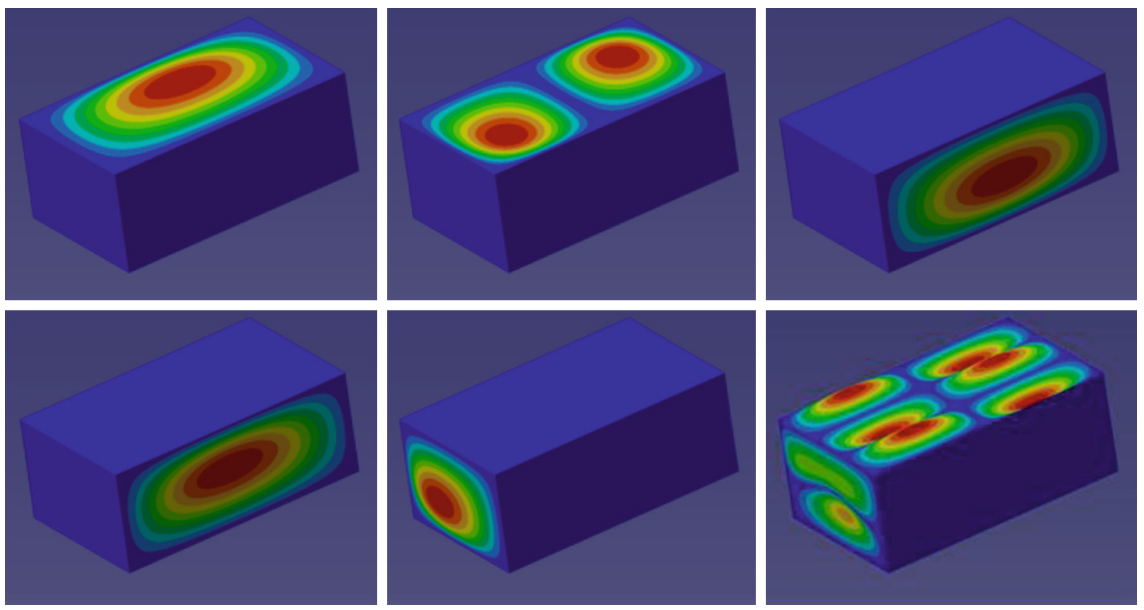

Fig. 2 The mode shapes, Mode 1, 2, 3, 4, 5 and 25 (a-f), of the box under different ambient temperature

Fig. 3 The acoustic response, the sound pressure level $\left(p_{\text {ref }}=2.0 \times 10^{-5} \mathrm{~Pa}\right)$ at the center point of the box

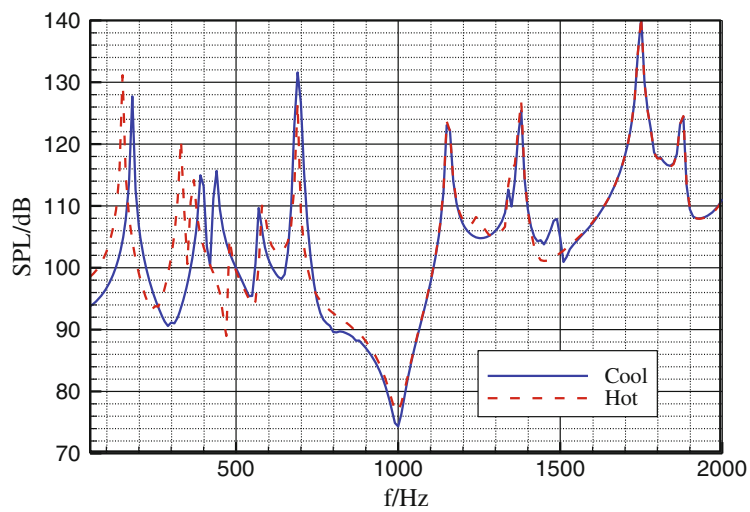

where $S P L_{\text {in }}$ is the sound pressure level of the incident sound wave, which is $140 \mathrm{~dB}$ (Fig. 4).

The result shows that, around every natural frequency of the structure, the sound transmission would appear a corresponding peak. The transmission loss of the 'hot' box is slightly less than that of the 'cool' one. However, in higher frequency bands, above about $1500 \mathrm{~Hz}$, such difference would disappear.

The acoustic response in the middle-' $Z$ ' section is given, as Fig. 5 shows. The sound wave transmits into the box; the acoustic field in the box appears welldistributed in the box, in the region near the wall, the sound pressure level decreases soon. 


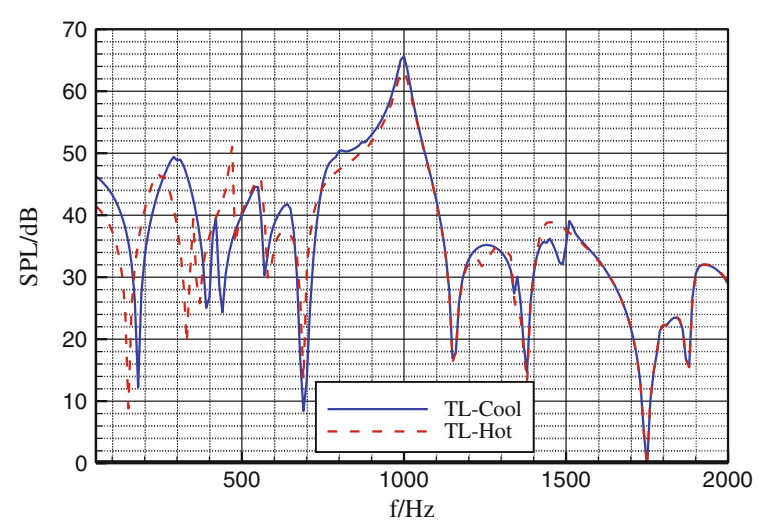

Fig. 4 The transmission loss, $T L_{(1)}$ of the box
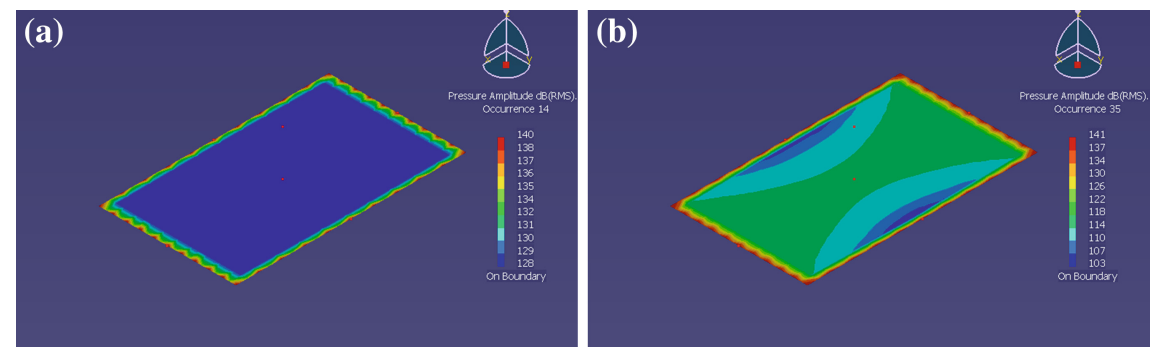

Fig. 5 The sound pressure level contour in the middle-' $Z$ ' section, the cool case at $180 \mathrm{~Hz}(\mathbf{a})$ and $430 \mathrm{~Hz}(\mathbf{b})$

Furthermore, the vibration response can be found, shown in Fig. 6. It is seen that the displacement response shape is similar with the natural mode shape at the same frequency, by comparing Figs. 6 and 2. Thus, the resonance effect was numerically simulated.

The sound power on the middle-' $Z$ ' section, $P_{\text {mid }}$, is obtained, thus the transmission loss $(T L)$ can be computed in another expression,

$$
T L_{(2)}=10 \log \left(P_{\text {in }} / P_{\text {mid }}\right), \text { where } P_{\text {in }}=\frac{p_{\text {rms }}^{2}}{\rho_{0} c} S
$$

and $S$ is the area of the top face of the box, and $P_{\text {in }}$ is the sound power of the incident sound wave. Figure 7 gives the sound power level and the transmission loss, $T L_{(2)}$, according to formula (6). The result shows that, the difference caused by temperature is not so much. However, the qualitative property of the transmission loss is the same, comparing with the result of $T L_{(1)}$, that is, the transmission loss of the 'hot' box is a little less than that of the 'cool' one and the valley frequencies is 

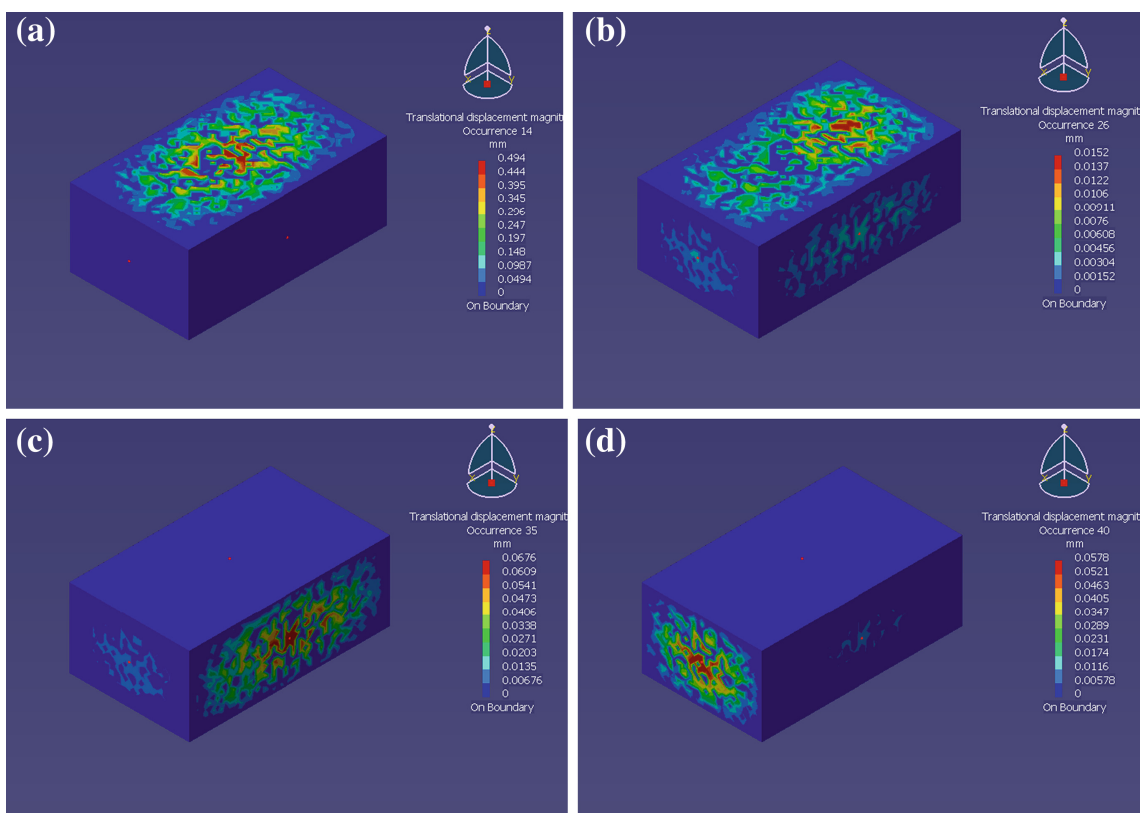

Fig. 6 The displacement response magnitude contour, on the inner surface of the 'cool' box, at $180 \mathrm{~Hz}(\mathbf{a}), 300 \mathrm{~Hz}(\mathbf{b}), 390 \mathrm{~Hz}(\mathbf{c})$ and $440 \mathrm{~Hz}(\mathbf{d})$

Fig. 7 The transmission loss, $T L_{(2)}$ (defined by Eq. 6) of the box

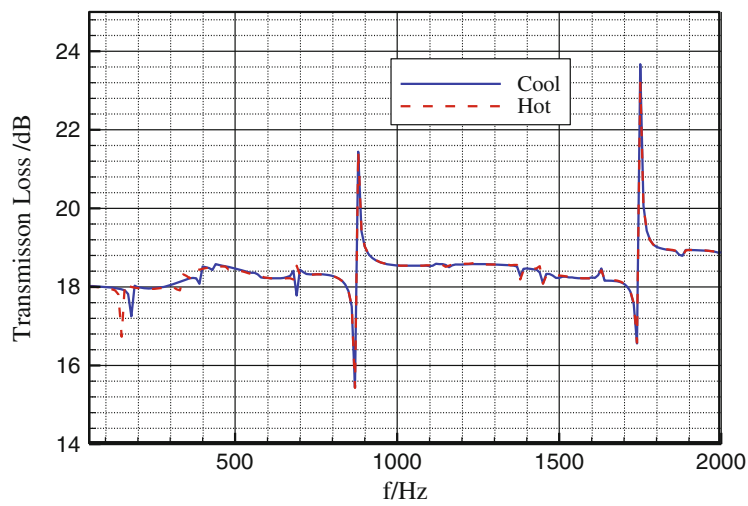

accord with the natural frequencies of the structure. Besides, in higher frequency bands, above $700 \mathrm{~Hz}$ around, the difference tends to disappear.

For the center point sound pressure level, as formula (5) defined, the transmission loss $\left(T L_{(1)}\right)$ is much greater than that defined by (6) $\left(T L_{(2)}\right)$, because the center point is a sensitive point, where the vibro-acoustic coupling is strong, than the points near the inner wall of the box and $T L_{(2)}$ reflects the total vibro-acoustic coupling effect. 


\section{Conclusion}

The paper gives a thermo-vibro-acoustic analysis of a thin-walled box, in order to investigate the multi-physics characteristics. The coupled FEM/BEM is implemented to analyze the structural and acoustic response. The effect of thermal environment is studied to consider both thermal stress and thermal softening of the materials, with only one plane acoustic wave exerted from the ambient. The sound pressure level of the middle-' $Z$ ' section, the transmission loss with two different definitions, and the vibration response on the inner surface are computed, to observe the vibro-acoustic characteristics. It is revealed that the acoustic responses around the low frequency would be obviously changed by the temperature elevation of the material, while such difference would disappear in the region of high frequency.

Acknowledgement The authors would like to thank the financial support by National Natural Science Foundation of China under Grant No. 11332011.

\section{References}

1. Hughes WO, McNelis ME (2002) Recent advances in vibro acoustics. NASA/TM-2002211697

2. Jeyaraj P, Padmanabhan C, Ganesan N (2008) Vibration and acoustic response of an isotropic plate in a thermal environment. J Vib Acoust 130(5):051005

3. Geng Q, Li Y (2012) Analysis of dynamic and acoustic radiation characters for a flat plate under thermal environments. Int J Appl Mech 4(3):1250028

4. Zhang ZX (ed) (1989) Handbook of enginery metal materials. Weapon Industry Press, Beijing (in Chinese) 